\title{
The Effect of Fibroblast Co-culture on In Vitro Maturation of Mouse Preantral Follicles
}

\author{
${ }^{\dagger}$ Chung-Hoon Kim${ }^{1}$, Yong-Pil Cheon ${ }^{2}$, You-Jeong Lee ${ }^{1}$, Kyung-Hee Lee ${ }^{1}$, Sung-Hoon Kim ${ }^{1}$, \\ Hee-Dong Chae ${ }^{1}$ and Byung-Moon Kang ${ }^{1}$ \\ ${ }^{1}$ Division of Reproductive Endocrinology and Infertility, Department of Obstetrics and Gynecology, \\ College of Medicine, University of Ulsan, Asan Medical Center, Seoul 138-736, Republic of Korea \\ ${ }^{2}$ Division of Development and Physiology, Department of Biology, Institute for Basic Science, \\ Sungshin Women's University, Seoul 136-742, Republic of Korea
}

\begin{abstract}
This study was performed to evaluate the effects of fibroblast co-culture on in vitro maturation (IVM) of prepubertal mouse preantral follicles. The intact preantral follicles were obtained from the ovaries of $12-14$ day old mice and these were cultured individually in $\alpha$-minimal essential medium ( $\alpha$-MEM) supplemented with $5 \%$ fetal bovine serum (FBS), $100 \mathrm{mIU} /$ recombinant follicle stimulating hormone (rFSH), 1\% insulin-transferrin-selenium, $100 \mu \mathrm{g} / \mathrm{ml}$ penicillin and 50 / streptomycin as base medium for 12 days. A total of 200 follicles were cultured in base medium co-cultured with mouse embryonic fibroblast (MEF) (MEF group) $(n=100)$ or only base medium as control group ( $n=100$ ). Survival rate of follicles on day 12 of culture were significantly higher in the MEF group of $90.0 \%$, compared with $77.0 \%$ of the control group ( $p=0.021$ ). Follicle diameters on day 6 and 8 of the culture period were significantly larger in the MEF group than those in the control group ( $p=0.021, p=0.007$, respectively). Estradiol levels in culture media on day 4, 6, 8, 10 and 12 of the culture period were significantly higher in the MEF group ( $p=0.043, p=0.021, p=0.006, p<0.001$ and $p=0.008$, retrospectively). Our data suggest that MEF cell co-culture on IVM of mouse preantral follicle increases survival rate and promotes follicular growth and steroid production.
\end{abstract}

Key words : Co-culture, In vitro maturation, Mouse preantral follicles, Mouse embryonic fibroblast

\section{INTRODUCTION}

Recent studies on in vitro maturation (IVM) of immature oocytes and follicles have focused on the development of preantral follicular culture systems, because preantral follicles are large potential source for in vitro studies of folliculogenesis. This preantral follicle culture system can be used not only for investigation of the effects of endogenous and exogenous factors on folliculogenesis (Sun et al., 2004) but also for long-term preservation of female germ cells (Mao et al., 2002).

Cellular interactions between ovarian germline and somatic cell components are crucial for follicular development (Eppig, 1991) and some compounds secreted from somatic cells have been reported to assist with the growth of preantral follicles (Tan et al., 2007). Therefore co-culture with somatic cells can be applied to improve in vitro development of oocytes and embryos (Haidari et al., 2008).

\footnotetext{
Manuscript received 29 August 2013, Received in revised form 4 September 2013, Accepted 12 September 2013

${ }^{\dagger}$ Corresponding Author : Chung-Hoon Kim, Division of Reproductive Endocrinology and Infertility, Department of Obstetrics and Gynecology, College of Medicine, University of Ulsan, Asan Medical Center, 88, Olympic-ro 43-gil, Songpa-gu, Seoul 138-736, Korea. Tel. : +82-2-3010-3639, Fax : +82-23010-6944, E-mail : chnkim@amc.seoul.kr

This is an Open Access article distributed under the terms of the Creative Commons Attribution Non-Commercial License(http://creativecommons.org/ licenses/by-nc/3.0) which permits unrestricted non-commercial use, distribution, and reproduction in any medium, provided the original work is properly cited.
} 
Use of somatic cells as a feeder layer in co-culture systems can improve embryo quality and increase the rate of embryo development into the blastocyst stage (Malekshah \& Moghadam, 2005). Somatic cells used in the co-culture system have been reported to produce certain promoting factors for embryonic development or remove embryo toxic materials from the culture medium (Hajializadeh et al., 2008) However, the use of a feeder layer for IVM of preantral follicles in the coculture system has received less attention.

Fibroblast cells are a major part of ovarian stromal cells and they secrete several cytokines such as leukemia inhibitory factor (LIF) and basic fibroblast growth factor (bFGF) that promote the ovarian follicle maturation (Demeestere et al., 2005; Hatoya et al., 2006). Angiogenesis is a critical component in the folliculogenesis and formation of ovarian corpora lutea. Of the many promoters for angiogenesis that have been identified, the most important factors appear to be vascular endothelial growth factors (VEGFs) and FGFs (Seghezzi et al., 1998; Berisha, 2001). FGFs are necessary for numerous biological processes and induce not only angiogenic activity but also mitogenic and chemotactic activity in the various cells and tissues (Rubin et al., 1989). Although the action mechanism of FGFs in the ovary has not been extensively explored, bFGF was found to be important in initiating follicular development and appears to be a primordial follicle-inducing factor (Nilsson et al., 2001). Therefore, co-culturing with mouse embryonic fibroblast (MEF) cells may promote preantral follicle maturation and we performed this study to evaluate MEF co-culture on in vitro maturation (IVM) of prepubertal mouse preantral follicles and their immature oocytes.

\section{MATERIAL AND METHODS}

\section{Animals and preantral follicle isolation}

Male and female ICR mice were housed and bred under a 12 hour light $/ 12$ hour dark regime at $22-24^{\circ} \mathrm{C}$. Postnatal 12-14 day-old female mice were sacrificed by cervical dislocation and their ovaries were isolated. Ovaries were immediately transferred to $\alpha$-minimal essential medium ( $\alpha$-MEM; Gibco, UK) supplemented with 5\% fetal bovine serum (FBS; Gibco, UK), 1\% insulin-transferrin-selenium (ITS; Gibco, UK), 100 / penicillin and 50 / streptomycin under mineral oil.

Preantral follicles from ovaries were mechanically isolated using a 29-gauge needle under stereomicroscope. Isolated follicles that were 120-150 in diameter and had 1 or 2 layers of granulosa cells, some adhering theca cells and centrally located round healthy oocyte were selected. All selected follicles were randomly divided into co-culture group cultured in basic medium with MEF (MEF group) and control group cultured in only basic medium. Then, isolated follicles were transferred to fresh culture medium.

\section{In vitro maturation}

The isolated preantral follicles were individually cultured in $60 \mathrm{~mm}$ petri dishes (Falcon, Becton Dickinson, Belgium) that contained 20 droplets of $\alpha$-MEM medium supplemented with 5\% FBS, $100 \mathrm{mIU} /$ recombinant follicle stimulating hormone (rFSH; Gonal-f, Merck-Serono, Geneva, Switzerland), 1\% ITS, $20 \mathrm{ng} /$ murine recombinant epidermal growth factor (rEGF; Sigma, Hamburg, Germany), 100 / penicillin and 50 / streptomycin as base medium in a humidified $5 \% \mathrm{CO}_{2}$ at $37^{\circ} \mathrm{C}$ under mineral oil for 12 days (Bishonga et al., 2001). A total of 200 follicles were cultured in base medium co-cultured with mouse embryonic fibroblast (MEF) (MEF group) ( $n=100)$ or only base medium as control group $(n=100)$.

Follicle diameter was measured using a precalibrated ocular micrometer at $100 \times$ magnifications every 48 hours from day 2 of the culture. The survival rate of the follicles was determined by evaluation of follicle morphology under an inverted microscope. Follicle containing an intact oocyte surrounded by granulosa cells attached to the culture dish was regarded as a survived follicle.

In every culture dishes, half of the medium was collected from each medium droplet every 48 hours and stored at 
$-20^{\circ} \mathrm{C}$ until analysis. Then, it was replaced by 15 of fresh pre-equlibrated medium.

\section{Preparation of MEF feeder layer}

MEFs were prepared according to Hatoya's method (Hatoya et al., 2006). Fetuses of mice were obtained from female ICR mice at days 12 to 13 of pregnancy and washed thoroughly with Dulbecco's phosphate-buffer saline (PBS). Fetal head and liver were removed and remaining fetal parts were cut into small pieces and cultured in a-MEM supplemented with 10\% FBS, 100 IU/ penicillin and $100 \mathrm{mg} /$ streptomycin in humidified $5 \% \mathrm{CO}_{2}$ at $37^{\circ} \mathrm{C}$. Primary fibroblasts were cultured until proliferated and confluent stage through two subsequent passages. For preparation of a feeder layer, MEF was inactivated by 10 / mitomycin C (Kyowa, Tokyo, Japan) for 3 hours in humidified $5 \% \mathrm{CO}_{2}$ at $37^{\circ} \mathrm{C}$ and washed five times in PBS. Subsequently, these cells were cryopreserved at $-80^{\circ} \mathrm{C}$ in a concentration of $10^{6}$ cells/ in $\alpha$-MEM supplemented with 60\% FBS, 100 IU/ penicillin, $100 \mathrm{mg} / \quad$ streptomycin and 10\% dimethyl sulfoxide (DMSO; ATCC, Manassas, Virginia, USA). Cryopreserved cells were thawed in warm water at $37^{\circ} \mathrm{C}$, cultured and plated at a density of $1 \times 10^{5}$ cells/ on the preceding day of the use.

\section{In vitro ovulation induction}

On day 12 of the culture, ovulation was induced by the addition of fresh medium supplemented with $1.5 \mathrm{IU} /$

human chorionic gonadotropin (hCG; Pregnyl, MSD, Griekenweg, The Netherlands) to each culture media.
The ovulated oocytes were observed under the inverted microscope after $20 \mathrm{hr}$ post induction (Olympux X70).

\section{Estradiol assessment}

Measurement of estradiol was performed by RIA using a Coat-A-Count Estradiol kit (Siemens Medical Solutions Diagnostics, Los Angeles, CA). Interassay and intraassay variances were less than $10 \%$ and $5 \%$, respectively.

\section{Statistical analysis}

Results are reported as means \pm standard deviations (SDs). The Chi-squared test, Fisher's exact test and Student's $t$-test were used, as appropriate, in comparisons. Statistical significance was defined as $P<0.05$. The SPSS statistical package for Windows, version 11.0 (SPSS Inc, Chicago, IL) was used for all analyses.

\section{RESULTS}

Survival rate of follicles on day 12 of culture were significantly higher in the MEF group of $90.0 \%$, compared with $77.0 \%$ of the control group $(p=0.021)$ (Table 1). Antrum formation rate and ovulation rate on day 12 of culture seemed to be higher in the MEF group, but these differences did not achieve the statistical significance (Table 1).

Follicle diameters on day $0,2,4,10$ and 12 of the culture period were similar between the two groups. However, follicle diameters on day 6 and 8 were significantly higher in the MEF group than those in the control group ( $p=0.021, p=0.007$, respectively) (Table 2). Estradiol

Table 1. Comparison of survival, antrum formation and ovulation rates of preantral follicles between the MEF cell co-culture and control groups

\begin{tabular}{lccc}
\hline & MEF group & Control group & $P$ \\
\hline No. of cultured preantral follicles & 100 & 100 & $77.0(77 / 100)$ \\
Survival rate $(\%)$ & $90.0(90 / 100)$ & $84.4(65 / 77)$ & 0.021 \\
Antrum formation rate & $88.9(80 / 90)$ & $80.5(62 / 77)$ & NS \\
Ovulation rate & $86.7(78 / 90)$ & NS \\
\hline
\end{tabular}

Values are means \pm standard deviations. MEF, mouse embryonic fibroblast; NS, not significant. 
Table 2. Comparison of follicle diameters ( ) between the MEF cell co-culture and control groups

\begin{tabular}{cccc}
\hline \hline Culture day & MEF group & Control group & $P$ \\
\hline Day 0 & $131.3 \pm 3.1$ & $133.7 \pm 4.0$ & NS \\
Day 2 & $160.5 \pm 13.8$ & $158.9 \pm 18.8$ & NS \\
Day 4 & $258.0 \pm 41.7$ & $237.9 \pm 48.3$ & NS \\
Day 6 & $377.9 \pm 78.0$ & $308.3 \pm 80.6$ & 0.021 \\
Day 8 & $473.6 \pm 71.4$ & $370.0 \pm 77.6$ & 0.007 \\
Day 10 & $488.7 \pm 66.8$ & $450.5 \pm 70.9$ & NS \\
Day 12 & $512.3 \pm 55.3$ & $488.3 \pm 60.2$ & NS \\
\hline
\end{tabular}

Values are means \pm standard deviations. MEF, mouse embryonic fibroblast; NS, not significant.

Table 3. Comparison of estradiol concentrations (pg/ ) of culture media during culture period of preantral follicles between the MEF cell co-culture and control groups

\begin{tabular}{ccrcc}
\hline Culture day & \multicolumn{1}{c}{ MEF group } & Control group & $P$ \\
\hline Day 4 & $55.8 \pm 8.3$ & $31.0 \pm 5.3$ & 0.043 \\
Day 6 & $258.5 \pm 32.3$ & $208.2 \pm 33.3$ & 0.021 \\
Day 8 & $988.0 \pm 67.2$ & $802.5 \pm 60.7$ & 0.006 \\
Day 10 & $2,120.7 \pm 115.9$ & $1,611.2 \pm 120.5$ & $<0.001$ \\
Day 12 & $3,845.3 \pm 235.8$ & $3,545.8 \pm 265.4$ & 0.008 \\
\hline
\end{tabular}

Values are means \pm standard deviations. MEF, mouse embryonic fibroblast; NS, not significant.

concentrations in culture media on day 4, 6, 8, 10 and 12 of the culture period were significantly higher in the MEF group ( $p=0.043, p=0.021, p=0.006, p<0.001$ and $p=$ 0.008 , retrospectively) (Table 3 ).

\section{DISCUSSION}

Preantral follicles are a large potential source of oocytes, and therefore the development of effective culture system for preantral follicle is very important. In the present study, the effects of co-culture with MEF cells were evaluated on the IVM of isolated preantral follicles from prepubertal mouse ovaries. Our results demonstrated that survival rate of follicles on day 12 of culture were significantly higher in the MEF cell co-culture group than in the control group. In addition, this study also showed that follicle diameters increased during the culture of isolated preantral follicles in both MEF cell co-culture and control groups and that these were significantly higher in the MEF group especially on day 6 and 8 of culture. These results suggest that the MEF cell co-culture used in the present study have the potential to improve the survival and promote the growth of preantral follicles, and the certain factors from MEF cells play a role as a proliferative factor for granulosa and theca cells. The most promising factor that acts as promoting factor for the growth of preantral follicles is bFGF. Nilsson et al. (2001) demonstrated that bFGF is localized to primordial and early developing follicles and it induces primordial follicle development and promote cell growth. Basic FGF may affect primordial follicle development by acting on oocytes, granulosa cells, theca cells or stromal interstitial cells (Shikone et al., 1992; Manova et al., 1993). The potential ability of bFGF for direct regulation of theca cell or ovarian stromal cell function has not been thoroughly investigated. Factors for controlling the initiation of primordial follicle development are essential for female reproduction. Initiation of primordial follicle development determines the number of follicles available for selection of dominant follicle. Therefore, the thorough understanding of bFGF and clinical application are important for the development of effective IVM system, controlled ovarian stimulation (COS) for poor responders and new method for fertility preservation.

Our results of this study showed that the MEF cell co-culture group had a higher growth rate especially on day 6 and 8 of the culture period and survival rate when compared to the control group. It has been known that FSH stimulates proliferation and differentiation of the granulosa cells and also induces FSH and luteinizing hormone (LH) receptors in the granulosa cells. On the other hand, the function of gonadotropins in follicular development are indirectly regulated by expression of kit ligand (KL) and fibroblast growth factors (FGF) which are secreted from fibroblast cells (McGee et al., 1999). Therefore, MEF cells may contribute to the growth and survivability of preantral follicles by providing growth 
factors such as bFGF and LIF (Hatoya et al., 2006).

In the present study, antrum formation and ovulation rate of follicles were higher in the MEF group than in the control group, but the differences were not statistically significant. Theses results may result from small numbers of preantral follicles that were used for the study. Therefore, larger and extended studies are needed to verify the effect of MEF cell co-culture on antrum formation and ovulation rates of preantral follicles.

Because steroid hormones may be important regulators of crucial changes in oocyte cytoplasm for normal fertilization, measurements of estradiol of follicle culture media can provide precise information on the functionality of the culture condition (Cortvrindt et al., 1998). As shown in Table 3, estradiol concentrations increased in a linear fashion up to day 12 of follicle culture in both MEF and control groups. Estradiol levels were significantly higher in the MEF cell co-culture group everytime estradiol concentrations of culture media were measured from the day 4 of culture period. This significant production of estradiol can result from the supportive effects of the fibroblast co-culture system on in vitro growth and maturation of preantral follicles. In this study, progesterone concentrations of culture media were not measured because preantral follicles were cultured only for 12 days, because progesterone production in cultured preantral follicles was started lately when compared with estradiol. Actually, Cortvrindt et al. has reported that progesterone was detected from day 8 of culture onward, and the highest levels were achieved on day 14 and 16 of the culture period (Cortvrindt et al., 1996).

In conclusion, MEF cell co-culture may have a beneficial effect on IVM of preantral follicle and improve survival and growth rates of preantral follicles. However, more research is required to confirm the effect of MEF cell co-culture on IVM of preantral follicles and improve the MEF co-culture system for preantral follicles.

\section{REFERENCES}

Berisha B, Amselgruber W, Kosmann M, Schams D, Einspanier R (2001) Expression, tissue concentration and localisation of some angiogenic factors during the final growth of bovine ovarian follicles. Exp Clin Endocrinol Diabetes 109(Suppl 1):67.

Bishonga C, Takashi Y, Katagiri S, Nagano M, Ishikawa A (2001) In vitro growth of ovarian peantral follicles and the capacity of their oocytes to develop to the blastocyst stage. J Vet Med Sci 63:619-624.

Cortvrindt R, Smitz J, Van Steirteghem AC (1996) In-vitro maturation, fertilization and embryo development of immature oocytes from early preantral follicles from prepubertal mice in a simplified culture system. Hum Reprod 11:2656-2666.

Cortvrindt R, Hu Y, Smitz Y (1998) Recombinant luteinizing hormone as a survival and differentiation factor increases oocyte maturation in recombinant follicle stimulating hormone-supplemented mouse preantral follicle culture. Hum Reprod 13:1292-1302.

Demeestere I, Centner J, Gervy C, Englert Y, Delbaere A (2005) Impact of various endocrine and paracrine factors on in vitro culture of preantral follicles in rodents. Reproduction 130:147-156.

Eppig JJ (1991) Intercommunication between mammalian oocytes and companion somatic cells. Bioassays 13: 569-574.

Haidari K, Salehnia M, Rezazadeh Valojerdi M (2008) The effect of leukemia inhibitory factor and coculture on the in vitro maturation and ultrastructure of vitrified and nonvitrified isolated mouse preantral follicles. Fertil Steril 90:2389-2397.

Hajializadeh N, Babaei H, Nematollahi-Mahani SN, Azizollahi S (2008) The development of mouse early embryos in vitro in fibroblasts and cumulus cells co-cultures supplemented with retinoic acid. Iranian J Vet Res 9:1-8.

Hatoya S, Sugiyama Y, Torii R, Wijewardana V, Kumagai D, Sugiura K (2006) Effect of co-culturing with embryonic fibroblasts on IVM, IVF and IVC of canine oocyte. Theriogenology 66:1083-1090. 
Malekshah AK, Moghadam AE (2005) Follicular fluid and cumulus cells synergistically improve mouse early embryo development in vitro. J Reprod Dev 51: 195-199.

Manova K, Huang EJ, Angeles M, De Leon V, Sanchez S, Pronovost SM, Besmer P, Bachvarova RF (1993) The expression pattern of the c-kit ligand in gonads of mice supports a role for the c-kit receptor in oocyte growth and in proliferation of spermatogonia. Dev Biol 157:85-99.

Mao J, Wu G, Smith MF, McCauley TC, Cantley TC, Prather RS (2002) Effects of culture medium, serum type and various concentrations of follicule stimulating hormone on porcine preantral follicular development and antrum formation in vitro. Biol Reprod 67: 1197-1203.

McGee EA, Chun SY, Lai S, He Y, Hsueh AJ (1999) Keratinocyte growth factor promotes the survival, growth and differentiation of preantral follicles. Fertil Steril 71:732-738.

Nilsson E, Parrott JA, Skinner MK (2001) Basic fibroblast growth factor induces primordial follicle development and initiates folliculogenesis. Mol Cell Endocrinol 175:123-130.
Rubin JS, Osada H, Finch PW, Taylor WG, Rudikoff S, Aaronson SA (1989) Purification and characterization of a newly identified growth factor specific for epithelial cells. Proc Natl Acad Sci USA 86:802-806.

Seghezzi G, Patel S, Ren CJ, Gualandris A, Pintucci G, Robbins ES, Shapiro RL, Galloway AC, Rifkin DB, Mignatti P (1998) Fibroblast growth factor-2 (FGF-2) induces vascular endothelial growth factor (VEGF) expression in the endothelial cells of forming capillaries: An autocrine mechanism contributing to angiogenesis. J Cell Biol 141:1659-1673.

Shikone T, Yamoto M, Nakano R (1992) Follicle stimulating hormone induces functional receptors for basic fibroblast growth factor in rat granulosa cells. Endocrinology 131:1063-1068.

Sun F, Betzendahl I, Shen Y, Cortvrindt R, Smitz J, Eichenlaub-Ritter U (2004) Preantral follicle culture as a novel in vitro assay in reproductive toxicology testing in mammalian oocytes. Mutagenesis 19:13-25. Tan XW, Ma SF, Yu JN, Zhang X, Lan GC, Liu XY (2007) Effects of species and cellular activity of oviductal epithelial cells on their dialogue with cocultured mouse embryos. Cell Tissue Res 327:55-66. 